\title{
Estudio de los cambios morfológicos en el yeyuno proximal después de hepatectomía parcial en la rata
}

\author{
M. B. Rodríguez, F. J. del Riego Tomás y C. Vaquero ${ }^{1}$ \\ Servicio de Cirugía General. Hospital General Río Carrión. Palencia. 'Servicio de Cirugía Vascular y Angiología. \\ Hospital Clínico Universitario. Valladolid
}

\section{RESUMEN}

Introducción: la hepatectomía parcial es una técnica quirúrgica que ha adquirido un gran auge en los últimos años, sus indicaciones quirúrgicas son cada vez más amplias, incluyendo el trasplante de donante vivo, principalmente en la infancia. Nuestro objetivo es estudiar los cambios morfológicos que se producen en el yeyuno proximal, porque el hígado y el intestino delgado se comportan como una unidad anatómica, funcional y metabólica.

Material y métodos: se han utilizado 24 ratas de la raza Wistar divididas en 2 grupos, el grupo control y el grupo experimental (30, 90 y 180 días). Se han estudiado los cambios que se originan en el yeyuno proximal después de una resección hepática parcial del 70\%, valorando 4 parámetros (grosor total de la pared, altura de la vellosidad, profundidad de la cripta y grosor de la vellosidad).

Resultados: se produce una elevación significativa del grosor total de la pared del yeyuno en el grupo experimental $(p<$ 0,001). La altura de la vellosidad se incrementa en el grupo experimental significativamente respecto al grupo control $(p<0,001)$. Se observa un aumento en el grosor de la vellosidad en el grupo experimental y una disminución en la profundidad de las criptas que no son estadísticamente significativos.

Conclusiones: la hepatectomía parcial del $70 \%$ produce unos cambios tróficos en el yeyuno proximal, que se mantienen a corto y largo plazo, ocasionando una hipertrofia de la pared y de las vellosidades.

Palabras clave: Yeyuno proximal. Hepatectomía parcial.

\begin{abstract}
Introduction: partial hepatectomy is being used with increasing frequency and wider surgical indications including living donor transplantation, particularly in children. Our aim was to study the morphologic changes that occur in the proximal jejunum as a result of the anatomic, functional, and metabolic unity of the liver and small intestine.
\end{abstract}

Material and methods: twenty-four Wistar rats were divided into 2 groups, a control group and an experimental group (30, 90 , and 180 days). Changes in the proximal jejunum after $70 \%$ partial liver resection were studied by evaluating 4 parameters (total wall width, villous height, crypt depth, and villous thickness).

Results: jejunal total wall width increased significantly in the experimental group $(p<0.001)$. Villous height increased significantly in the experimental group as compared to the control group ( $p<0.001)$. Villous thickness increased, and crypt depth decreased in the experimental group, but these changes were not statistically significant.

Conclusions: partial hepatectomy (70\%) originated trophic changes in the proximal jejunum that persisted in the short and long term, giving rise to parietal and villous hypertrophy.

Key words: Proximal jejunum. Partial hepatectomy.

Rodríguez MB, del Riego Tomás FJ, Vaquero C. Estudio de los cambios morfológicos en el yeyuno proximal después de hepatectomía parcial en la rata. Rev Esp Enferm Dig 2008; 100: 615-618.

\section{INTRODUCCIÓN}

La resección hepática parcial es una técnica quirúrgica que se ha desarrollado enormemente en las últimas dos décadas, gracias al mejor conocimiento de la anatomía

Recibido: 24-04-08

Aceptado: 17-06-08.

Correspondencia: María Belén Rodríguez Sanz. Servicio de Cirugía General. Hospital General Río Carrión. Avda. de los Donantes de Sangre, s/n. 34005 Palencia. e-mail: brosanz@yahoo.es quirúrgica y al progreso de los medios tecnológicos, permitiendo ampliar cada vez más las indicaciones quirúrgicas, incluyendo el trasplante de hígado de donante vivo.

El hígado y el intestino funcionan como una unidad, una resección hepática amplia produce una serie de alteraciones enzimáticas y metabólicas que pueden afectar al intestino delgado y otros órganos.

Diversos estudios experimentales demostraron que después de la pérdida de volumen hepático hay una redistribución de la capacidad fagocítica (1) y una sobreactivación de los macrófagos extrahepáticos que ocasionan 
fallos de la barrera intestinal y una deficiencia contra la infección (2); todo ello depende de varios factores como el tipo de resección, hemorragia intraoperatoria y tiempo quirúrgico; estos tres agentes fueron descritos por Andersson y Yanaga $(3,4)$. Estos factores favorecen los cambios en la permeabilidad intestinal que conduce al sobrecrecimiento bacteriano y traslocación bacteriana, concepto este que fue descrito por Berg (5).

La mucosa del intestino delgado puede ser modificada por diversos factores como el tipo de nutrición, las secreciones biliopancreáticas, hormonas entéricas, intervenciones quirúrgicas, etc. $(6,7)$.

Estos hechos nos han conducido a la realización de este trabajo, siendo nuestro objetivo estudiar los posibles cambios morfológicos que se originen en el yeyuno proximal después de efectuar una hepatectomía del 70\%.

\section{MATERIAL Y MÉTODOS}

Se han empleado 24 ratas de la raza Wistar de un peso aproximado de 200-240 gramos, procedentes del Animalario de la Facultad de Medicina de Valladolid que se encuentran en unas condiciones de habitabilidad estándar adaptadas a las normativas dictadas por la Directiva Europea de noviembre de 1986 y de la Legislación Española de marzo de 1988.

Los animales fueron divididos en dos grupos, un grupo control $(n=6)$ a los cuales se les practicó una sección del yeyuno proximal y un grupo experimental $(n=18)$ a los que se les realiza una hepatectomía parcial del 70\%; posteriormente se efectúa la sección del yeyuno proximal a los 30, 90 y 180 días $(n=6,6$ y 6).

La técnica de la hepatectomía parcial se realizó mediante anestesia general con clorhidrato de ketamina. Se practicó una laparotomía media, extracción de los lóbulos hepáticos lateral izquierdo y del lóbulo medio, se identifican los vasos sanguíneos y se seccionan, posteriormente se procede a una ligadura y sección de ambos lóbulos lo que clásicamente constituyen la denominada hepatectomía parcial del $70 \%$. A continuación se procede al cierre de la laparotomía y se colocan a los animales en armarios de recuperación a una temperatura y humedad adecuadas con reintroducción precoz de su alimentación habitual.

Transcurridos 30, 90 y 180 días respectivamente en cada subgrupo del grupo experimental, las ratas nuevamente se someten a una anestesia general con clorhidrato de ketamina, relaparotomía media, identificación del ángulo de Treizt, se miden unos $10 \mathrm{~cm}$ y se seccionan $7 \mathrm{~cm}$. Se finaliza con el sacrificio del animal.

En el grupo control sólo se realiza la sección de $7 \mathrm{~cm}$ del yeyuno proximal procediendo de la misma manera que en el grupo experimental.

Seguidamente se efectúa un estudio macroscópico de las muestras obtenidas. El estudio histológico se realizó mediante fijación en formol al 10\%, inclusión en parafina y tinción con hematoxilina-eosina.
Se realizó un estudio morfométrico de las vellosidades del yeyuno evaluando los parámetros del grosor total de la pared, altura de la vellosidad, profundidad de la cripta y grosor de la vellosidad.

Los datos obtenidos se sometieron a un análisis estadístico, realizando un estudio descriptivo con el cálculo de la media y su desviación estándar. Se hizo un análisis de bondad de ajuste a la normalidad con el test de Shapiro-Wilks. Cuando la distribución de la variable es normal, se realizó un análisis de la varianza con contrastes $a$ posteriori con el test de Sheffe, si la distribución no sigue la ley normal se utilizó el test de Kruskall-Wallis.

\section{RESULTADOS}

Macroscópicamente no se encontró ninguna alteración en el yeyuno del grupo experimental respecto al grupo control.

El grosor total de la pared del yeyuno a los 30 días $(\mathrm{n}=$ 6) aumentó en un $53,78 \%$, a los 90 días $(\mathrm{n}=6)$ se incrementó en un $37,45 \%$, y a los 180 días $(\mathrm{n}=6)$ en un $59,76 \%$. Este incremento es estadísticamente significativo $(\mathrm{p}<0,001)$ respecto al grupo control y se mantiene en el tiempo (Tabla I).

Tabla I. Valores medios del grosor total de la pared del yeyuno proximal y su desviación estándar

\begin{tabular}{lcc}
\hline Grupos & $X \pm D S$ & Significación estadística \\
\hline Control & $6,2750 \pm 0,8595$ & \\
30 días & $9,6500 \pm 1,2685$ & $<0,001$ \\
90 días & $8,6250 \pm 0,5889$ & $<0,001$ \\
180 días & $10,0250 \pm 1,2341$ & $<0,001$ \\
\hline
\end{tabular}

X: valor medio de los datos obtenidos medidos en unidades píxel; DS: desviación estándar de los datos.

La altura de la vellosidad a los 30 días $(\mathrm{n}=6)$ aumenta en un $148,83 \%$, a los 90 días $(n=6)$ en un $146,12 \%$, y a los 180 días $(n=6)$ aumenta en un $181,78 \%$. Estas diferencias son estadísticamente significativas respecto a las ratas no hepatectomizadas, para $\mathrm{p}<0,001$ (Tabla II).

El grosor de las vellosidades a los 30 días $(n=6)$ en las ratas que han sido sometidas a una hepatectomía parcial del $70 \%$ han crecido en un $11,44 \%$, a los 90 días $(\mathrm{n}=$ 6) aumentan en un $13,92 \%$, y a los 180 días $(n=6)$ en un $13,43 \%$. Esta diferencia respecto al grupo control no es estadísticamente significativa (Tabla III).

La profundidad de las criptas en ratas hepatectomizadas a los 30 días $(\mathrm{n}=6)$ disminuye en un 15,20\%, a los 90 días en un $35,18 \%$, y a los 180 días desciende en un $17,19 \%$. Estas diferencias no son estadísticamente significativas en relación a las ratas no hepatectomizadas (Tabla IV). 
Tabla II. Valores medios de la altura de la vellosidad del yeyuno proximal y su desviación estándar

\begin{tabular}{lcc}
\hline Grupos & $X \pm D S$ & Significación estadística \\
\hline Control & $2,1500 \pm 0,2387$ & \\
30 días & $5,3500 \pm 0,9391$ & $<0,001$ \\
90 días & $5,2917 \pm 0,5995$ & $<0,001$ \\
180 días & $6,0583 \pm 0,6383$ & $<0,001$ \\
\hline
\end{tabular}

X: valor medio de los datos obtenidos medidos en unidades píxel; DS: desviación estándar de los datos.

Tabla III. Valores medios del grosor de la vellosidad del yeyuno proximal y su desviación estándar

\begin{tabular}{lcc}
\hline Grupos & $X \pm D S$ & Significación estadística \\
\hline Control & $1,6750 \pm 0,2788$ & \\
30 días & $1,8667 \pm 0,3737$ & NS \\
90 días & $1,9083 \pm 0,2710$ & NS \\
180 días & $1,900 \pm 0,5874$ & NS \\
\hline X: valor medio de los datos obtenidos medidos en unidades píxel; DS: desviación estándar de los datos.
\end{tabular}

Tabla IV. Valores medios de la profundidad de la cripta del yeyuno proximal y su desviación estándar

\begin{tabular}{lcc}
\hline Grupos & $X \pm D S$ & Significación estadística \\
\hline Control & $2,8417 \pm 0,7067$ & \\
30 días & $2,4667 \pm 0,5654$ & NS \\
90 días & $2,100 \pm 0,2191$ & NS \\
180 días & $2,4250 \pm 0,4298$ & NS \\
\hline
\end{tabular}

X: valor medio de los datos obtenidos medidos en unidades píxel; DS: desviación estándar de los datos.

\section{DISCUSIÓN}

Se han realizado numerosos estudios experimentales sobre las alteraciones que la hepatectomía parcial produce sobre el intestino delgado como cambios enzimáticos, sobrecrecimiento bacteriano, traslocación bacteriana, etc., así como los fenómenos de regeneración hepática ocasionados en el hígado remanente, hechos fisiopatológicos específicos de la resección hepática o modificaciones enzimáticas y metabólicas.

La regeneración hepática fue observada por primera vez en 1894 por Ponfick en perros. Este concepto conlleva una restauración de la estructura anatómica original después de una hepatectomía parcial, asocia dos fenómenos: uno de hipertrofia y otro de hiperplasia celular que se definen como un incremento en el número y tamaño de las células. Estudios realizados por Rabes y cols. (8) determinaron que los hepatocitos de la rata proliferan por lo menos una vez en el transcurso de las 24-36 horas después de la resección hepática parcial, terminando la regeneración completa en unos 20 días. Se regula por estricto control homeostático (9) y es dependiente de cambios hormonales.

La hepatectomía parcial en la rata es una técnica quirúrgica sencilla, pero puede haber complicaciones. Estas dependen de una serie de factores descritos por Anders- son y Yanaga $(3,4)$ tales como tiempo operatorio, hemorragia intraoperatoria, tipo de resección y procedimientos quirúrgicos; en nuestro trabajo el tiempo operatorio oscila entre 10 y 15 minutos y la hemorragia operatoria es mínima, de unos 10-20 ml. La tasa de mortalidad es del $1-4 \%$ y la de morbilidad del $25 \%$ (10). En nuestro estudio la mortalidad ha sido del $0 \%$ y no se ha observado morbilidad aparente.

Esta cirugía predispone a infecciones bacterianas, sepsis, sobrecremiento y traslocación bacteriana a nivel intestinal que depende de la cantidad de hígado resecado (11), produciendo alteraciones en la mucosa de la barrera intestinal.

La resección hepática produce un fallo de la barrera intestinal cuyos factores responsables más importantes son la disminución de la circulación sanguínea en la mucosa del intestino fundamentalmente en el íleon distal, excesiva activación de los macrófagos intestinales y cambios en la permeabilidad intestinal que ocasiona edema tisular, disfunción epitelial e invasión bacteriana (12).

Se han descrito una serie de factores que modifican la mucosa intestinal como el tipo de nutrición, secreciones biliopancreáticas, hormonas entéricas, intervenciones quirúrgicas, shock hemorrágico, quemaduras, sistema nervioso autónomo, etc. Se ha demostrado que la nutrición enteral tiene efectos tróficos sobre la mucosa intestinal, disminuyendo los disturbios de la flora intestinal y las complicaciones sépticas, mientras que la nutrición parenteral y el ayuno intensifican las lesiones que se producen en la mucosa del intestino delgado. Las secreciones biliopancreáticas y las hormonas entéricas ejercen una acción trófica (13).

Alverdy (14) e Illig (15) en sus estudios observaron que la nutrición parenteral produce atrofia intestinal que favorece el sobrecrecimiento y la traslocación bacteriana.

Nuestros animales, una vez efectuada la resección hepática fueron introducidos en armarios de recuperación a una temperatura y humedad adecuadas, ya que se ha demostrado que la prevención de la hipotermia que se produce después de una laparotomía por evaporación y de la hipoglucemia mejora la supervivencia de las ratas; así mismo se introdujo de forma precoz la nutrición enteral y su alimentación habitual debido a la acción trófica que ejercen sobre la mucosa intestinal como nos demuestran Qui y cols. (16) en su trabajo previniéndolos contra la sepsis. De esta manera en nuestro trabajo hemos obtenido una supervivencia global del $100 \%$ de nuestros animales.

En la rata, el tamaño de las vellosidades intestinales disminuye gradualmente desde el duodeno hasta el íleon, siendo de una altura en este último de menos de la mitad de su tamaño en el duodeno (17).

Como hemos mencionado anteriormente, la bilis y las secreciones pancreáticas inducen efectos tróficos sobre la mucosa intestinal como nos lo demuestra Altmann en 1971 (18), realizando un trasplante de la papila duodenal a segmentos ileales con crecimiento en la altura de las vellosidades en este nivel, con un crecimiento entre el 60-70\%, 
en nuestro estudio se produce una elevación media del $150 \%$.

Otros autores como Riepe (19) manifiestan que la colonización y el sobrecrecimiento bacteriano en el segmento proximal del intestino por bacterias entéricas puede ser factor patogénico en la atrofia de las vellosidades, en nuestro estudio se produce una hipertrofia de las mismas.

Podemos concluir que una hepatectomía parcial del $70 \%$ en la rata induce una hipertrofia de la pared del intestino delgado en sus segmentos proximales, así como una hipertrofia de las vellosidades, sin ejercer modificaciones ni en la profundidad de las criptas ni en el grosor de la vellosidad.

\section{BIBLIOGRAFÍA}

1. Camañas Sanz A. Regeneración hepática: aspectos morfológicos del hígado durante los 4 primeros días posthepatectomía. Rev Esp Enferm Dig 1981; 60 (6): 571-8.

2. Wang XD, Ar'Rajab A, Andersson R, Soltesz V, Wang W, Svensson $\mathrm{M}$, et al. The influence of surgically induced acute liver failure on the intestine in the rat. Scand J Gastroenterol 1993; 28: 31-40.

3. Andersson R, Sarela A, Tranberg KG, Bengmark S. Intrabdominal abscesses formation after major liver resection. Acta Chir Scand 1990; 156: 707-10.

4. Yanaga K, Kanematsu T, Sugimachi K Takenaka K. Intraperitoneal septic complications after hepatectomy. Ann Surch 1986; 203: 14852 .

5. Berg RD. Translocation of indigenous bacteria from the intestinal tract. In: Hentges DJ, editor. Human intestinal microflora in health and disease. New York: Academic Press; 1983. p. 333-52.

6. Baena Hidalgo A, Carpio González M, García Ruiz C, Carrera Moreno G, Fuentes García S, Carpio Molinos M. Estudio morfoenzimático de la mucosa yeyunal normal en la rata Wistar. Rev Esp Enferm Dig 1984; 66 (6): 473-80.

7. Wang XD, Pärsson H, Soltesz V, Johansson K, Andersson R. Bacterial translocation and intestinal capillary permeability following major liver resection in the rat. J Surg Res 1995; 58: 351-8.

8. Rabes HM. Kinetics of hepatocellular proliferation as a function of the liver. In hepatotrophyc factors. CIBA Foundation Symposium, No. SS. Amsterdam: Elsevier; 1978. p. 31-40.

9. Bucher NLR. Regeneration of mammalian liver. Int Rev Cytology 1963; 15: 245-300.

10. Thompson HH, Tompkins RK, Longmire WP, Jr. Major hepatic resection: a 25 years experience. Ann Surg 1983; 197: 375-88.

11. Wang XD, Andersson R, Soltesz V, Bengmark S. Bacterial translocation after major hepatectomy in patients and rats. Arch Surg 1992; 127: 1101-6.

12. Wang XD, Andersson R, Soltesz V Guo W, Bengmark S. Water-soluble ethylhydroxethyl cellulose prevents bacterial translocation induced by major liver resection in the rat. Ann Surg 1993; 217: 155-67.

13. Dowling RH. Small bowel adaptation and its regulation. In: Polak SM, editor. Structure of the gut. London: Glaxo Group Research Limited; 1982. p. 369-90.

14. Alverdy J, Chi JS, Sheldon GF. The effect of parenteral nutrition on gastrointestinal immunity. The importance of enteral stimulation. Ann Surg 1985; 202: 681-6.

15. Illig KA, Ryan CK, Hardy DJ. Total parenteral nutrition induced changes in gut mucosal function: atrophy alone is not the tissue. Surg 1992; 112: 631-7.

16. Qui JG, Delany HM, Teh EL, Freundlich L, Gliedman ML, Steinberg LL, et al. Contrasting effects of identical nutrients given parenterally or enterally after $70 \%$ hepatectomy. Bacterial translocation. Nutrition 1997; $13: 431-7$.

17. Altmann GG, Enesco M. Cell number as a measure of distribution and renewal of epithelial cells in the small intestine of growing and adult rats. Am J Anat 1967; 121: 319-36.

18. Altmann GG. Influence of bile and pancreatic secretions on size of the intestinal villi in the rat. Am J Anat 1971; 132: 167-78.

19. Riepe SP, Goidstein J, Alpers DH. Effect of secreted bacteroides proteases on human intestinal brush border hydrolases. J Clin Invest 1980; 66: 314-22. 